\title{
Finite-Time Synchronization of Complex Multilinks Networks with Perturbations and Time-Varying Delay Based on Nonlinear Adaptive Controller
}

\author{
Lixiang Li $\mathbb{D},{ }^{1,2}$ Qingbiao Liu, ${ }^{2}$ and Tao $\mathrm{Li}^{3}$ \\ ${ }^{1}$ School of Computer Science and Technology, Henan Polytechnic University, Jiaozuo 454003, Henan, China \\ ${ }^{2}$ Information Security Center, State Key Laboratory of Networking and Switching Technology, \\ Beijing University of Posts and Telecommunications, Beijing 100876, China \\ ${ }^{3}$ College of Computer Science, Sichuan University, Sichuan 610065, China
}

Correspondence should be addressed to Lixiang Li; li_lixiang2006@163.com

Received 17 December 2018; Accepted 31 March 2019; Published 16 April 2019

Academic Editor: Sabri Arik

Copyright @ 2019 Lixiang Li et al. This is an open access article distributed under the Creative Commons Attribution License, which permits unrestricted use, distribution, and reproduction in any medium, provided the original work is properly cited.

\begin{abstract}
This paper utilizes nonlinear adaptive feedback controller to make the complex multilinks networks with perturbations and timevarying delays achieve the finite-time synchronization. By designing nonlinear controllers, we use suitable Lyapunov functions and sufficient conditions to guarantee the finite-time synchronization between the drive system and the response system in terms of adaptive control. Several novel and useful finite-time synchronization criteria are accurately derived based on linear matrix inequality, Kronecker product, inequality analytical technique, and finite-time stability theory. Finally, numerical examples are given to demonstrate the validity and the effectiveness of our theoretical results.
\end{abstract}

\section{Introduction}

With the development of human social networks, the theory of complex networks has penetrated many fields such as mathematical science, life science, engineering science, and even social science [1-6]. Their applications have also been involved in nature, engineering technology, biological technology, and human society [7-11]. There is no doubt that complex network has become one of the most concerning and challenging scientific frontiers in scientific researches on complex systems. Many scholars especially have paid wide attention to the stability and the synchronization control of various complex networks [12-14]. Plotnikov et al. studied the synchronization control of the delay-coupled neural networks with heterogeneous nodes through the adaptively tuned time-delayed coupling [12]. Sorrentino et al. described a method to find and analyze all of the possible cluster synchronization patterns in a Laplacian-coupled network by applying the methods of computational group theory to dynamically equivalent networks [13]. Zheng et al. introduced the synchronization of uncertain coupling recurrent dynamical neural networks with time-varying delays based on the adaptive controller and parameters update rules [14].

The control of nonlinear systems is also one of the focus issues discussed in both academical and industrial fields. Many different control methods have been applied into the control of nonlinear systems, such as impulsive control [15], pinning control [16], intermittent control [17], and adaptive control [18]. Among these control schemes, the adaptive control is a control scheme that can track the change of system states. It can change the dynamic states of the system and modify the parameters of the controller at any time, which can maintain a good control effect. At present, some researches have emerged on the synchronization of complex dynamical networks via the adaptive control [19-22]. Delellis et al. used the decentralized adaptive strategy to achieve the synchronization of complex networks [19]. Based on the local information of node dynamics, Yu et al. investigated the synchronization of complex networks by the distributed adaptive control [20]. Nian utilized the adaptive control to make the complex network with uncertain boundary achieve the coupling synchronization and he proposed a concept about 


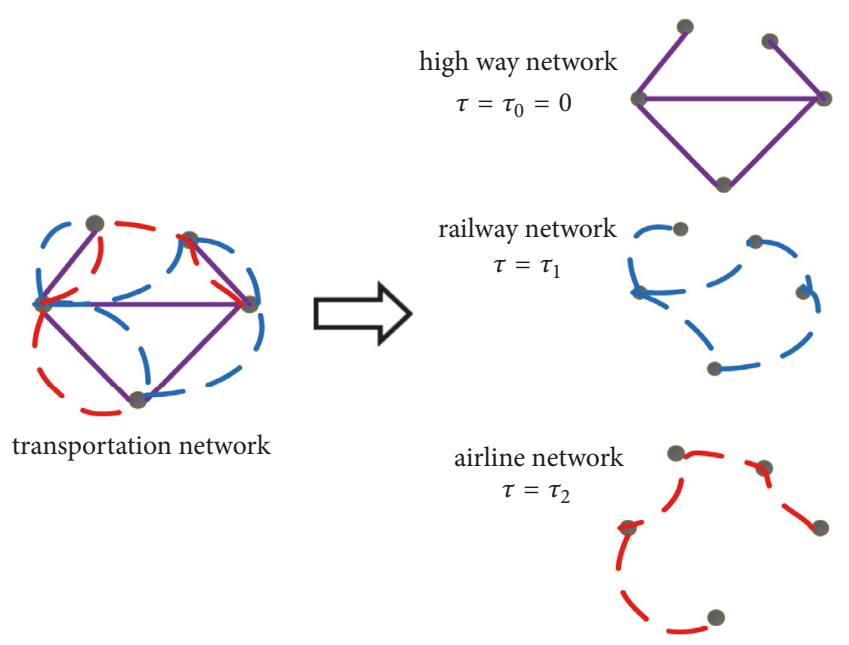

FIgURE 1: Transportation network and its division. According to different transmission speeds of different paths, the transportation network can be split into highway network, railway network, and airline network.

the asymptotic stability [21]. Lu et al. introduced the robust adaptive synchronization of general dynamical networks with multiple delays and uncertainties by employing the robust adaptive control principle and the Lyapunov stability theory [22].

The complex networks with multilinks exist widely in human society such as ecological network, transportation network, and relationship network [23-25]. A complex multilinks network is composed of the subnetworks with various properties, and its multiple edges between any two nodes may have different properties. What is more, these subnetworks are also complex networks. For example, the transmission speed is different among highway network, railway network, and airline network in the transportation network, which is shown in Figure 1. An individual goes to the same place from the same starting point, and there may exist different time delays on different paths. Thus, the transportation network can be split into three subnetworks by different time delays, i.e., the highway network, the railway network, and the airline network. In most cases, the time delay is also the time-varying delay.

Recently, some scholars have studied the adaptive synchronization of complex dynamical networks with multilinks [26-29]. Peng et al. made two complex networks with multilinks and different structures achieve the synchronization by adaptive control, Lyapunov stability theory, and some hypotheses [26]. Jiang et al. studied the local and global synchronizations of complex networks with coupling delays and multilinks based on some simple controllers with the updated feedback strength [27]. Wang et al. gave a definition of similar nodes for the first time and investigated the synchronization of complex multilinks networks with similar nodes via analyzing the minimal similarity of similar nodes and some adaptive synchronization criteria [28]. In most practical situations, it usually requires that complex networks should achieve the synchronization in the finite time. Zheng et al. discussed the finite-time synchronization of complex multilinks dynamical networks with or without internal time delays via intermittent controls [29]. Furthermore, there must exist some uncertain or inevitable factors in practical systems such as external perturbations and uncertain parameters. However, the previous researches mainly focused on the synchronization of complex multilinks network via adaptive control. Few researches on the synchronization of complex networks involve finite time, perturbations, and time-varying delay which are very important in practical applications. Therefore, the research on the finite-time synchronization control in complex multilinks networks with perturbations and time-varying delay is more representative, realistic, and valuable in some engineering applications.

Motivated by the above discussions, we study the finitetime synchronization of complex multilinks networks with stochastic perturbation and time-varying delay. The contributions of this paper are presented as follows:

(1) This is the first time to investigate the finite-time synchronization problem of the complex multilinks networks with perturbations and time delay, including constant time delay and time-varying delay.

(2) We design the adaptive nonlinear feedback controller to guarantee the synchronization of drive-response networks in a finite time by using the finite-time stability theory and several inequality analytical techniques.

(3) For complex multilinks networks with perturbations and time-varying delay, we construct novel criteria about the finite-time synchronization between these networks via utilizing linear matrix inequality.

(4) In order to illustrate the effectiveness and the feasibility of our theoretical results, we harness different network models, such as E-R random networks [30], B-A scale-free networks [31], and small-world networks [32], in numerical simulations.

The rest of this paper is organized as follows. Section 2 presents the network model and some preliminaries. In Section 3, some criteria are obtained for the finite-time synchronization by rigorous derivation. Section 4 gives numerical simulation results. Finally, Section 5 concludes this paper.

\section{Network Model and Preliminaries}

This paper discusses the model of complex multilinks network consisting of $N$ nodes with $m$ kinds of properties. The state equation of this model is given as

$$
\begin{gathered}
d x_{i}(t)=\left[h\left(x_{i}(t)\right)+\sum_{j=1}^{N} c_{(0) i j} x_{j}(t)\right. \\
+\sum_{j=1}^{N} c_{(1) i j} x_{j}\left(t-\tau_{1}(t)\right)+\cdots \\
\left.+\sum_{j=1}^{N} c_{(m-1) i j} x_{j}\left(t-\tau_{m-1}(t)\right)\right] d t
\end{gathered}
$$




$$
\begin{aligned}
& +\sigma\left(t, x_{i}(t)\right) d \varphi(t)=\left[h\left(x_{i}(t)\right)\right. \\
& \left.+\sum_{j=1}^{N} c_{(0) i j} x_{j}(t)+\sum_{k=1}^{m-1} \sum_{j=1}^{N} c_{(k) i j} x_{j}\left(t-\tau_{k}(t)\right)\right] d t \\
& +\sigma\left(x_{i}(t), t\right) d \varphi(t), \quad i=1,2, \ldots, N
\end{aligned}
$$

where $x_{i}(t)=\left(x_{i 1}(t), x_{i 2}(t), \ldots, x_{i n}(t)\right)^{T} \in R^{n}$ is the drive state vector of the $i$ th node, $h\left(x_{i}(t)\right)$ is a smooth nonlinear vector function, $\tau_{k}(t)(k=0,1, \ldots, m-1)$ is the time-varying delay of the $k$ th subnetwork, and $\tau_{0}=0$ means the subnetwork with zero time-varying delay. $C_{k}=$ $\left(c_{(k) i j}\right)_{N \times N}(k=0,1, \ldots, m-1)$ represents the topological structure of the $k$ th subnetwork and it is a Laplace matrix, where $c_{(k) i j}$ is defined as $c_{(k) i j}>0$ if there is an edge from node $i$ to node $j(i \neq j)$; otherwise, $c_{(k) i j}=c_{(k) j i}=0$. The entry $c_{(k) i j}$ indicates whether there is an edge from node $i$ to node $j$ in the $k$ th subnetwork. The connection strength of the edge can be represented by

$$
c_{(k) i i}=-\sum_{j=1, j \neq i}^{N} \mathcal{c}_{(k) i j}, \quad i=1,2 \ldots, N .
$$

In the perturbation term of (1), $\sigma: R^{n} \times R^{+} \longrightarrow R^{n \times m}$ is the noise intensity function, $\varphi(t)=\left(\varphi_{1}(t), \varphi_{2}(t), \ldots, \varphi_{m}(t)\right)^{T}$ is an $m$-dimensional Brownian motion defined on a complete probability space $(\Omega, F, P)$ satisfying $E[d \varphi(t)]=0$, $E\left\{[d \varphi(t)]^{2}\right\}=d t$, where $E[\cdot]$ is the mathematical expectation, and every two elements are statistically independent. Hence, for the noise intensity, we can deduce that $E[\sigma(x, t) d \varphi(t)]=0$ and $E\left\{[\sigma(x, t) d \varphi(t)]^{T}[\sigma(x, t) d \varphi(t)]\right\}=$ $\operatorname{trace}\left[\sigma(x, t)^{T} \sigma(x, t)\right] d t$.

In system (1), some subnetworks may be random networks, and some may be scale-free networks or small-world networks. In this paper, we regard (1) as the driver system, and the corresponding response system is given as

$$
\begin{array}{r}
d y_{i}(t)=\left[h\left(y_{i}(t)\right)+\sum_{j=1}^{N} c_{(0) i j} y_{j}(t)\right. \\
\left.+\sum_{k=1}^{m-1} \sum_{j=1}^{N} c_{(k) i j} y_{j}\left(t-\tau_{k}(t)\right)\right] d t \\
+\sigma\left(y_{i}(t), t\right) d \varphi(t)+u_{i}(t) d t
\end{array}
$$

$$
i=1,2, \ldots, N
$$

where $y_{i}(t)=\left(y_{i 1}(t), y_{i 2}(t), \ldots, y_{i n}(t)\right)^{T} \in R^{n}$ is the response state vector of the $i$ th node and $u(t)=\left(u_{1}(t), u_{2}(t), \ldots\right.$, $\left.u_{N}(t)\right)^{T}$ is an adaptive nonlinear controller to be designed. The synchronization errors between system (1) and system (3) are defined as $e_{i}(t)=y_{i}(t)-x_{i}(t)(1 \leq i \leq N)$.

To obtain main results of this paper, we have the following definitions, assumptions, and lemmas.
Definition 1. System (1) and system (3) can achieve the finitetime synchronization in mean square for suitable controller $u_{i}(t)$ and any given initial conditions, if there exists a constant $T_{s}>0$ such that

$$
\lim _{t \rightarrow T_{s}} E\left\|e_{i}(t)\right\|^{2}=0, \quad i=1,2, \ldots, N,
$$

where $E\left\|e_{i}(t)\right\|=0$ for $t>T_{s},\|\cdot\|$ stands for the Euclidean vector norm, and $T_{s}$ is the theoretical finite time on the synchronization.

Assumption 2. For any $x, y \in \mathbb{R}^{n}$, there exists a nonnegative constant $\omega$, which makes $h$ satisfy the following inequality:

$$
\|h(y)-h(x)\| \leq \omega\|y-x\| .
$$

Assumption 3. There exists a nonnegative constant $\delta$ such that

$$
\begin{aligned}
& \operatorname{trace}\left\{\left[\sigma\left(y_{i}, t\right)-\sigma\left(x_{i}, t\right)\right]^{T} \times\left[\sigma\left(y_{j}, t\right)-\sigma\left(x_{j}, t\right)\right]\right\} \\
& \leq \delta\left(\left\|y_{i}-x_{i}\right\|^{2}+\left\|y_{j}-x_{j}\right\|^{2}\right)
\end{aligned}
$$

where $\operatorname{trace}(\cdot)$ stands for the trace of the matrix, and $i, j=$ $1,2, \ldots, N$.

Assumption 4. There exists a differential function $\tau(t)$ which satisfies $0 \leq \dot{\tau}(t) \leq \varepsilon<1$. It is apparent that this assumption is justified if $\tau(t)$ is a constant.

Lemma 5 (see [33]). If there exists a continuous positivedefinite function $V(t)$ such that the following inequality holds,

$$
\dot{V}(t) \leq-\alpha V^{\eta}(t), \quad \forall t \geq t_{0}, V\left(t_{0}\right) \geq 0,
$$

where $\alpha>0$ and $0<\eta<1$ are two constants, then, for any given $t_{0}, V(t)$ satisfies the following inequality:

$$
V^{1-\eta}(t) \leq V^{1-\eta}\left(t_{0}\right)-\alpha(1-\eta)\left(t-t_{0}\right), \quad t_{0} \leq t \leq t_{1}
$$

and $V(t) \equiv 0, \forall t \geq t_{1}$ with $t_{1}$ given by $t_{1}=t_{0}+V^{1-\eta}\left(t_{0}\right) / \alpha(1-$ $\eta)$.

Lemma 6 (see [34]). For any $\beta>0, a, b \in \mathbb{R}^{n}$, there exists the inequality

$$
2 a^{T} b \leq \beta a^{T} a+\beta^{-1} b^{T} b .
$$

Lemma 7 (see [35]). If $b_{1}, b_{2}, \ldots, b_{n}$ are positive numbers and $0<r<p$, then

$$
\left(\sum_{i=1}^{n} b_{i}^{p}\right)^{1 / p} \leq\left(\sum_{i=1}^{n} b_{i}^{r}\right)^{1 / r}
$$

Lemma 8 (see [35]). If $b_{1}, b_{2}, \ldots, b_{n}$ and $0<p \leq 1$, then

$$
\left(\sum_{i=1}^{n} b_{i}\right)^{p} \leq \sum_{i=1}^{n} b_{i}^{p}
$$


Lemma 9 (see [36]). Consider the system

$$
\begin{aligned}
d x= & h(x(t), x(t-\tau), t) d t \\
& +h(x(t), x(t-\tau), t) d \varphi(t),
\end{aligned}
$$

and suppose that there exists a $C^{2}$ function $V: R^{n} \longrightarrow R_{+}$and $\mu_{1}, \mu_{2}, \mu_{3} \in C\left(R^{n}, R_{+}\right)$such that

$$
\begin{aligned}
\mu_{1}(\|x\|) & \leq V(x, t) \leq \mu_{2}(\|x\|), \\
L V(t) & =-\mu_{3}(\|x\|),
\end{aligned}
$$

where $L$ is the weak infinitesimal operators of stochastic processes. Then the equilibrium $x=0$ is stochastically asymptotically stable. Especially, if $\mu_{1}, \mu_{2}, \mu_{3} \in C\left(R^{n}, R_{+}\right)$, then the equilibrium $x=0$ is globally stochastically asymptotically stable.

Notations: $R^{n}$ denotes the $n$-dimensional Euclidean space. The superscript $T$ denotes the matrix transposition. The notation $A \geq B$ (respectively, $A>B$ ) means that $A-$ $B$ is positive semidefinite (respectively, positive-definite). The matrices of this paper are assumed to have compatible dimensions if not explicitly stated.

\section{Main Results}

In this section, we define a finite-time synchronization criteria via adaptive nonlinear feedback controller $u(t)=\left(u_{1}(t)\right.$, $\left.u_{2}(t), \ldots, u_{N}(t)\right)^{T}$, which is defined as follows:

$$
\begin{aligned}
& u_{i}(t)=-d_{i}(t) e_{i}(t)-p \operatorname{sign}\left(e_{i}(t)\right)-q\left(\frac{e_{i}(t)}{\left\|e_{i}(t)\right\|^{2}}\right) \\
& \cdot\left(\frac{1}{\xi_{i}}\left(d_{i}(t)-v\right)^{2}\right)^{1 / 2}-r\left(\frac{e_{i}(t)}{\left\|e_{i}(t)\right\|^{2}}\right) \\
& \cdot \sum_{k=1}^{m-1}\left(\int_{t-\tau_{k}(t)}^{t} \frac{1}{1-\varepsilon} e_{i}^{T}(s) e_{i}(s) d s\right)^{1 / 2}, \\
& \dot{d}_{i}(t)=\xi_{i} e_{i}^{T}(t) e_{i}(t)
\end{aligned}
$$

where $\xi_{i}$ is a positive constant for $1 \leq i \leq N, v$ is a constant, $p, q$, and $r$ are positive constants, $\varepsilon$ is a positive constant which satisfies $0 \leq \dot{\tau}(t) \leq \varepsilon<1$ under Assumption 4, and sign $(\cdot)$ is the sign function which is defined as

$$
\operatorname{sign}(x)= \begin{cases}-1, & \text { if } x<0, \\ 0, & \text { if } x=0, \\ 1, & \text { if } x>0,\end{cases}
$$

and $\operatorname{sign}\left(e_{i}(t)\right)=\left(\operatorname{sign}\left(e_{1}(t)\right), \operatorname{sign}\left(e_{2}(t)\right), \ldots, \operatorname{sign}\left(e_{n}(t)\right)\right)^{T}$.

Remark 10 (see [29]). If $e_{i}(t)=0$, let $e_{i}(t) /\left\|e_{i}(t)\right\|^{2}=0$.
According to the adaptive controller (14), the error of the dynamical system can be derived as follows:

$$
\begin{aligned}
& d e_{i}(t)=\left[h\left(y_{i}(t)\right)-h\left(x_{i}(t)\right)+\sum_{j=1}^{N} c_{(0) i j} e_{j}(t)\right. \\
& \left.+\sum_{k=1}^{m-1} \sum_{j=1}^{N} c_{(k) i j} e_{j}\left(t-\tau_{k}(t)\right)\right] d t \\
& +\sigma\left(y_{i}(t), t\right) d \varphi(t)-\sigma\left(x_{i}(t), t\right) d \varphi(t) \\
& +u_{i}(t) d t, \quad i=1,2, \ldots, N .
\end{aligned}
$$

We have the following results about the driver system (1), the response system (3), and the controller (14).

Theorem 11. Systems (1) and (3) can realize the finite-time synchronization via the nonlinear adaptive feedback controller (14), if there exists a constant $v$ such that the following condition holds:

$$
\begin{aligned}
& \left(\omega-\nu+\frac{1}{2}+\frac{m-1}{2(1-\varepsilon)}+\delta\right) \otimes I_{N}+C_{0}+\frac{1}{2} \sum_{k=1}^{m-1} C_{k}^{T} C_{k} \\
& \quad \leq 0
\end{aligned}
$$

where $\otimes$ is the Kronecker product, $\omega, \delta$, and $\varepsilon$ are positive constants under Assumptions 2-4, $\varepsilon$ satisfies $0 \leq \dot{\tau}(t) \leq \varepsilon<1$, $I_{N}$ is an $N \times N$ identity matrix, and $C_{0}$ and $C_{k}(k=1, \ldots, m-1)$ are the Laplace matrices previously mentioned.

The finite time of realizing the synchronization between system (1) and system (3) under the controller (14) is given as

$$
T_{s}=\frac{2 V^{1 / 2}(0)}{\sqrt{2} \alpha}
$$

where $\alpha=\min \{p, q, r\}$ and $V(0)$ is the following initial condition:

$$
\begin{aligned}
V(0)= & \frac{1}{2} \sum_{i=1}^{N} e_{i}^{T}(0) e_{i}(0)+\frac{1}{2} \sum_{i=1}^{N} \frac{1}{\xi_{i}}\left(d_{i}(0)-\nu\right)^{2} \\
& +\frac{1}{2(1-\varepsilon)} \sum_{k=1}^{m-1} \sum_{i=1}^{N} \int_{-\tau_{k}(t)}^{0} e_{i}^{T}(s) e_{i}(s) d s .
\end{aligned}
$$

Proof. Construct the following Lyapunov function:

$$
\begin{aligned}
V(t)= & \frac{1}{2} \sum_{i=1}^{N} e_{i}^{T}(t) e_{i}(t)+\frac{1}{2} \sum_{i=1}^{N} \frac{1}{\xi_{i}}\left(d_{i}(t)-v\right)^{2} \\
& +\frac{1}{2(1-\varepsilon)} \sum_{k=1}^{m-1} \sum_{i=1}^{N} \int_{t-\tau_{k}(t)}^{t} e_{i}^{T}(s) e_{i}(s) d s .
\end{aligned}
$$


$L$ is the weak infinitesimal operators of stochastic processes; then we can get

$$
\begin{aligned}
& L V(t)=\sum_{i=1}^{N} e_{i}^{T}(t)\left[h\left(y_{i}(t)\right)-h\left(x_{i}(t)\right)+\sum_{j=1}^{N} c_{(0) i j} e_{j}(t)\right. \\
& \left.+\sum_{k=1}^{m-1} \sum_{j=1}^{N} c_{(k) i j} e_{j}\left(t-\tau_{k}(t)\right)+u_{i}(t)\right]+\sum_{i=1}^{N} \frac{1}{\xi_{i}}\left(d_{i}(t)\right. \\
& -v) \dot{d}_{i}(t)+\frac{1}{2(1-\varepsilon)} \\
& \quad \cdot \sum_{k=1}^{m-1} \sum_{i=1}^{N}\left(\left(e_{i}^{T}(t) e_{i}(t)\right)-\frac{\left(1-\dot{\tau}_{k}(t)\right)}{2(1-\varepsilon)}\right. \\
& \quad \cdot \sum_{k=1}^{m-1} \sum_{i=1}^{N}\left(e_{i}^{T}\left(t-\tau_{k}(t)\right) e_{i}\left(t-\tau_{k}(t)\right)\right)+\frac{1}{2} \\
& . \sum_{i=1}^{N} \operatorname{trace}\left\{\left[\sigma\left(y_{i}(t), t\right)-\sigma\left(x_{i}(t), t\right)\right]^{T}\right. \\
& \left.\quad \times\left[\sigma\left(y_{i}(t), t\right)-\sigma\left(x_{i}(t), t\right)\right]\right\} .
\end{aligned}
$$

Based on Assumption 3, it can be obtained that

$$
\begin{aligned}
& \frac{1}{2} \sum_{i=1}^{N} \text { trace }\left\{\left[\sigma\left(y_{i}(t), t\right)-\sigma\left(x_{i}(t), t\right)\right]^{T}\right. \\
& \left.\quad \times\left[\sigma\left(y_{i}(t), t\right)-\sigma\left(x_{i}(t), t\right)\right]\right\} \leq \frac{1}{2} \\
& \cdot \sum_{i=1}^{N}\left(\delta\left\|y_{i}(t)-x_{i}(t)\right\|^{2}+\delta\left\|y_{i}(t)-x_{i}(t)\right\|^{2}\right) \\
& =\delta\left\|e_{i}(t)\right\|^{2}=\delta e_{i}^{T}(t) e_{i}(t) .
\end{aligned}
$$

From Assumption 4, we have

$$
1-\varepsilon \leq 1-\dot{\tau}_{k}(t)
$$

that is,

$$
\frac{1}{2} \leq \frac{\left(1-\dot{\tau}_{k}(t)\right)}{2(1-\varepsilon)} .
$$

According to Assumption 2 and substituting (22)-(24) and $u_{i}(t)$ into (21), it follows that

$$
\begin{gathered}
L V(t) \leq \sum_{i=1}^{N} e_{i}^{T}(t) \omega e_{i}(t)+\sum_{i=1}^{N} \sum_{j=1}^{N} c_{(0) i j} e_{i}^{T}(t) e_{j}(t) \\
+\sum_{i=1}^{N} \sum_{j=1}^{N} \sum_{k=1}^{m-1} c_{(k) i j} e_{i}^{T}(t) e_{j}\left(t-\tau_{k}(t)\right) \\
-\sum_{i=1}^{N} d_{i}(t) e_{i}^{T}(t) e_{i}(t)-p \sum_{i=1}^{N} e_{i}^{T}(t) \operatorname{sign}\left(e_{i}(t)\right) \\
-q \sum_{i=1}^{N}\left(\frac{e_{i}^{T}(t) e_{i}(t)}{\left\|e_{i}(t)\right\|^{2}}\right)\left(\frac{1}{\xi_{i}}\left(d_{i}(t)-v\right)^{2}\right)^{1 / 2}
\end{gathered}
$$

$$
\begin{aligned}
& -r \sum_{i=1}^{N}\left(\frac{e_{i}^{T}(t) e_{i}(t)}{\left\|e_{i}(t)\right\|^{2}}\right) \\
& \cdot \sum_{k=1}^{m-1}\left(\int_{t-\tau_{k}(t)}^{t} \frac{1}{1-\varepsilon} e_{i}^{T}(s) e_{i}(s) d s\right)^{1 / 2} \\
& +\sum_{i=1}^{N}\left(d_{i}(t)-v\right) e_{i}^{T}(t) e_{i}(t)+\frac{m-1}{2(1-\varepsilon)} \\
& \cdot \sum_{i=1}^{N} e_{i}^{T}(t) e_{i}(t)-\frac{1}{2} \\
& \cdot \sum_{i=1}^{N} \sum_{k=1}^{m-1} e_{i}^{T}\left(t-\tau_{k}(t)\right) e_{i}\left(t-\tau_{k}(t)\right)+\delta e_{i}^{T}(t) e_{i}(t) .
\end{aligned}
$$

Based on Lemma 6 and $\left(e_{i}^{T}(t) e_{i}(t) /\left\|e_{i}(t)\right\|^{2}\right)=1$, one obtains

$$
\begin{aligned}
L V(t) \leq & \sum_{i=1}^{N} e_{i}^{T}(t) \omega e_{i}(t)+\sum_{i=1}^{N} \sum_{j=1}^{N} c_{(0) i j} e_{i}^{T}(t) e_{j}(t) \\
& +\frac{1}{2} \sum_{i=1}^{N} \sum_{j=1}^{N} \sum_{k=1}^{m-1} e_{i}^{T}(t)\left(\left(c_{(k) i j}^{2}\right)\right) e_{i}(t) \\
& -p \sum_{i=1}^{N}\left\|e_{i}(t)\right\|-q \sum_{i=1}^{N}\left(\frac{1}{\xi_{i}}\left(d_{i}(t)-v\right)^{2}\right)^{1 / 2} \\
& -r \sum_{i=1}^{N} \sum_{k=1}^{m-1}\left(\int_{t-\tau_{k}(t)}^{t} \frac{1}{1-\varepsilon} e_{i}^{T}(s) e_{i}(s) d s\right)^{1 / 2} \\
& -\sum_{i=1}^{N} v e_{i}^{T}(t) e_{i}(t)+\frac{1}{2} \sum_{i=1}^{N} e_{i}^{T}(t) e_{i}(t) \\
& +\frac{m-1}{2(1-\varepsilon)} \sum_{i=1}^{N} e_{i}^{T}(t) e_{i}(t)+\delta e_{i}^{T}(t) e_{i}(t)
\end{aligned}
$$

Let $e(t)=\left[e_{1}(t), e_{2}(t), \ldots, e_{N}(t)\right]$, and according to condition (17), we get

$$
\begin{aligned}
& L V(t) \leq e^{T}(t)\left[\left(\omega-v+\frac{1}{2}+\frac{m-1}{2(1-\varepsilon)}+\delta\right) \otimes I_{N}\right. \\
& \left.+C_{0}+\frac{1}{2} \sum_{k=1}^{m-1} C_{k}^{T} C_{k}\right] e(t)-p \sum_{i=1}^{N}\left\|e_{i}(t)\right\| \\
& -q \sum_{i=1}^{N}\left(\frac{1}{\xi_{i}}\left(d_{i}(t)-v\right)^{2}\right)^{1 / 2} \\
& \quad-r \sum_{i=1}^{N} \sum_{k=1}^{m-1}\left(\int_{t-\tau_{k}(t)}^{t} \frac{1}{1-\varepsilon} e_{i}^{T}(s) e_{i}(s) d s\right)^{1 / 2} .
\end{aligned}
$$


According to Lemma 7, we can notice that

$$
\left(\sum_{i=1}^{N}\left\|e_{i}(t)\right\|^{2}\right)^{1 / 2} \leq\left(\sum_{i=1}^{N}\left\|e_{i}(t)\right\|^{1}\right)^{1} .
$$

Thus, we have

$$
\begin{aligned}
-p \sum_{i=1}^{N}\left\|e_{i}(t)\right\| & =-\sqrt{2} p\left(\sum_{i=1}^{N} \frac{1}{\sqrt{2}}\left\|e_{i}(t)\right\|\right)^{1} \\
& \leq-\sqrt{2} p\left(\sum_{i=1}^{N} \frac{1}{2}\left\|e_{i}(t)\right\|^{2}\right)^{1 / 2} \\
& =-\sqrt{2} p\left(\frac{1}{2} \sum_{i=1}^{N} e_{i}^{T}(t) e_{i}(t)\right)^{1 / 2} .
\end{aligned}
$$

Based on Lemma 8, we obtain the following inequalities:

$$
\begin{aligned}
& -q \sum_{i=1}^{N}\left(\frac{1}{\xi_{i}}\left(d_{i}(t)-v\right)^{2}\right)^{1 / 2} \\
& =-\sqrt{2} q \sum_{i=1}^{N}\left(\frac{1}{2 \xi_{i}}\left(d_{i}(t)-v\right)^{2}\right)^{1 / 2} \\
& \leq-\sqrt{2} q\left(\frac{1}{2} \sum_{i=1}^{N} \frac{1}{\xi_{i}}\left(d_{i}(t)-v\right)^{2}\right)^{1 / 2} . \\
& -r \sum_{i=1}^{N} \sum_{k=1}^{m-1}\left(\int_{t-\tau_{k}(t)}^{t} \frac{1}{1-\varepsilon} e_{i}^{T}(s) e_{i}(s) d s\right)^{1 / 2} \\
& =-\sqrt{2} r \sum_{i=1}^{N} \sum_{k=1}^{m-1}\left(\frac{1}{2} \int_{t-\tau_{k}(t)}^{t} \frac{1}{1-\varepsilon} e_{i}^{T}(s) e_{i}(s) d s\right)^{1 / 2} \\
& \leq-\sqrt{2} r\left(\frac{1}{2(1-\varepsilon)} \sum_{i=1}^{N} \sum_{k=1}^{m-1} \int_{t-\tau_{k}(t)}^{t} e_{i}^{T}(s) e_{i}(s) d s\right)^{1 / 2} .
\end{aligned}
$$

Substituting (29)-(31) into (27) and according to Lemmas 7 and 8 , we get

$$
\begin{aligned}
& L V(t) \leq e^{T}(t)\left[\left(\omega_{1}-v+\frac{1}{2}+\frac{m-1}{2(1-\varepsilon)}\right) \otimes I_{N}+C_{0}\right. \\
& \left.+\frac{1}{2} \sum_{k=1}^{m-1} C_{k}^{T} C_{k}\right] e(t)-\sqrt{2} p\left(\frac{1}{2} \sum_{i=1}^{N} e_{i}(t)^{T} e_{i}(t)\right)^{1 / 2} \\
& -\sqrt{2} q\left(\frac{1}{2} \sum_{i=1}^{N} \frac{1}{\xi_{i}}\left(d_{i}(t)-v\right)^{2}\right)^{1 / 2} \\
& \quad-\sqrt{2} r\left(\frac{1}{2(1-\varepsilon)} \sum_{i=1}^{N} \sum_{k=1}^{m-1} \int_{t-\tau_{k}(t)}^{t} e_{i}^{T}(s) e_{i}(s) d s\right)^{1 / 2} .
\end{aligned}
$$

Due to $\alpha=\min \{p, q, r\}$, it can be deduced that

$$
\begin{aligned}
& L V(t) \leq e^{T}(t)\left[\left(\omega-v+\frac{1}{2}+\frac{m-1}{2(1-\varepsilon)}+\delta\right) \otimes I_{N}\right. \\
& \left.+C_{0}+\frac{1}{2} \sum_{k=1}^{m-1} C_{k}^{T} C_{k}\right] e(t) \\
& -\sqrt{2} \alpha\left[\left(\frac{1}{2} \sum_{i=1}^{N} e_{i}^{T}(t) e_{i}(t)\right)^{1 / 2}\right. \\
& +\left(\frac{1}{2} \sum_{i=1}^{N} \frac{1}{\xi_{i}}\left(d_{i}(t)-v\right)^{2}\right)^{1 / 2} \\
& \left.+\left(\frac{1}{2(1-\varepsilon)} \sum_{i=1}^{N} \sum_{k=1}^{m-1} \int_{t-\tau_{k}(t)}^{t} e_{i}^{T}(s) e_{i}(s) d s\right)^{1 / 2}\right] .
\end{aligned}
$$

By Lemma 8, it can be obtained that

$$
\begin{aligned}
& L V(t) \leq e^{T}(t)\left[\left(\omega-v+\frac{1}{2}+\frac{m-1}{2(1-\varepsilon)}+\delta\right) \otimes I_{N}\right. \\
& \left.+C_{0}+\frac{1}{2} \sum_{k=1}^{m-1} C_{k}^{T} C_{k}\right] e(t)-\sqrt{2} \alpha\left[\frac{1}{2} \sum_{i=1}^{N} e_{i}^{T}(t) e_{i}(t)\right. \\
& +\frac{1}{2} \sum_{i=1}^{N} \frac{1}{\xi_{i}}\left(d_{i}(t)-v\right)^{2} \\
& \left.+\frac{1}{2(1-\varepsilon)} \sum_{i=1}^{N} \sum_{k=1}^{m-1} \int_{t-\tau_{k}(t)}^{t} e_{i}^{T}(s) e_{i}(s) d s\right]^{1 / 2} \\
& \quad=e^{T}(t)\left[\left(\omega-v+\frac{1}{2}+\frac{m-1}{2(1-\varepsilon)}+\delta\right) \otimes I_{N}+C_{0}\right. \\
& \left.+\frac{1}{2} \sum_{k=1}^{m-1} C_{k}^{T} C_{k}\right] e(t)-\sqrt{2} \alpha V^{1 / 2}(t) .
\end{aligned}
$$

If (17) holds, then we have

$$
L V(t) \leq-\sqrt{2} \alpha V^{1 / 2}(t) .
$$

From Itô's formula, we obtain that

$$
E V(t)-E V\left(t_{0}\right)=E \int_{t_{0}}^{t} L V(s) d(s) .
$$

From the definition of $V(t)$, there exists a positive constant $\gamma$ such that

$$
\begin{aligned}
\gamma E\|e(t)\|^{2} & \leq E V(t) \leq E V\left(t_{0}\right)+E \int_{t_{0}}^{t} L V(s) d(s) \\
& \leq E V\left(t_{0}\right)-\sqrt{2} \alpha E \int_{t_{0}}^{t} V^{1 / 2}(s) d s .
\end{aligned}
$$


According to Lemma 9, it is easy to conclude that the driver network (1) and the response network (3) achieve the finite-time synchronization in the mean square.

Using Lemma 5 and letting $t_{0}=0, \eta=1 / 2$, the driver system (1) and the response system (3) can realize the synchronization in the following finite time:

$$
T_{s}=t_{0}+\frac{V^{1-\eta}\left(t_{0}\right)}{\sqrt{2} \alpha(1-\eta)}=\frac{2 V^{1 / 2}(0)}{\sqrt{2} \alpha},
$$

where

$$
\begin{aligned}
V(0)= & \frac{1}{2} \sum_{i=1}^{N} e_{i}^{T}(0) e_{i}(0)+\frac{1}{2} \sum_{i=1}^{N} \frac{1}{\xi_{i}}\left(d_{i}(0)-\nu\right)^{2} \\
& +\frac{1}{2(1-\varepsilon)} \sum_{k=1}^{m-1} \sum_{i=1}^{N} \int_{-\tau_{k}(t)}^{0} e_{i}^{T}(s) e_{i}(s) d s .
\end{aligned}
$$

Thus, we complete the proof of Theorem 11.

Remark 12. According to Lemma 5 and Theorem 11, we can conclude that $T_{s}$ is the theoretical time to achieve the synchronization between the driver system (1) and the response system (3). Actually, the time may be shorter than the theoretical result in (38).

When $\tau_{k}(t)$ is a constant time delay $\tau_{k}(k=1,2, \ldots, m-1)$ where $\tau_{k}>0$, the driver system (1) and the response system (3) become the following forms:

$$
\begin{aligned}
& d x_{i}(t)=\left[h\left(x_{i}(t)\right)+\sum_{j=1}^{N} c_{(0) i j} x_{j}(t)\right. \\
& \left.+\sum_{k=1}^{m-1} \sum_{j=1}^{N} c_{(k) i j} x_{j}\left(t-\tau_{k}\right)\right] d t \\
& +\sigma\left(x_{i}(t), t\right) d \varphi(t), \quad i=1,2, \ldots, N, \\
& d y_{i}(t)=\left[h\left(y_{i}(t)\right)+\sum_{j=1}^{N} c_{(0) i j} y_{j}(t)\right. \\
& \left.+\sum_{k=1}^{m-1} \sum_{j=1}^{N} c_{(k) i j} y_{j}\left(t-\tau_{k}\right)\right] d t+\sigma\left(y_{i}(t), t\right) d \varphi(t) \\
& +u_{i}(t) d t, \quad i=1,2, \ldots, N .
\end{aligned}
$$

The nonlinear adaptive controller $u(t)=\left(u_{1}(t), u_{2}(t), \ldots\right.$, $\left.u_{N}(t)\right)^{T}$ will be changed as follows:

$$
\begin{aligned}
u_{i}(t) & \\
= & -d_{i}(t) e_{i}(t)-p \operatorname{sign}\left(e_{i}(t)\right) \\
& -q\left(\frac{e_{i}(t)}{\left\|e_{i}(t)\right\|^{2}}\right)\left(\frac{1}{\xi_{i}}\left(d_{i}(t)-v\right)^{2}\right)^{1 / 2}
\end{aligned}
$$

$$
\begin{array}{r}
-r\left(\frac{e_{i}(t)}{\left\|e_{i}(t)\right\|^{2}}\right) \sum_{k=1}^{m-1}\left(\int_{t-\tau_{k}}^{t} e_{i}^{T}(s) e_{i}(s) d s\right)^{1 / 2}, \\
\dot{d}_{i}(t)=\xi_{i} e_{i}^{T}(t) e_{i}(t), \\
i=1,2, \ldots, N,
\end{array}
$$

where all the parameters are the same as those in Theorem 11 except the time delay.

According to the adaptive controller (42), the error of the dynamical system can be derived as follows:

$$
\begin{array}{r}
d e_{i}(t)=\left[h\left(y_{i}(t)\right)-h\left(x_{i}(t)\right)+\sum_{j=1}^{N} c_{(0) i j} e_{j}(t)\right. \\
\left.+\sum_{k=1}^{m-1} \sum_{j=1}^{N} c_{(k) i j} e_{j}\left(t-\tau_{k}\right)\right] d t+\sigma\left(y_{i}(t), t\right) d \varphi(t) \\
-\sigma\left(x_{i}(t), t\right) d \varphi(t)+u_{i}(t) d t, \quad \\
\quad i=1,2, \ldots, N .
\end{array}
$$

Corollary 13. Systems (40) and (41) with multilinks can realize the finite-time synchronization via the adaptive controller (42) if there exists a constant $v$ such that the following condition holds:

$$
\left(\omega-\nu+\frac{m}{2}+\delta\right) \otimes I_{N}+C_{0}+\frac{1}{2} \sum_{k=1}^{m-1} C_{k}^{T} C_{k} \leq 0,
$$

where all the parameters are the same as those in Theorem 11.

Then system (40) and system (41) can realize the finite-time synchronization under the controller (42) in the finite time as follows:

$$
T_{s}=\frac{2 V^{1 / 2}(0)}{\sqrt{2} \alpha}
$$

where $\alpha=\min \{p, q, r\}$ and $V(0)$ is the following initial condition:

$$
\begin{aligned}
V(0)= & \frac{1}{2} \sum_{i=1}^{N} e_{i}^{T}(0) e_{i}(0)+\frac{1}{2} \sum_{i=1}^{N} \frac{1}{\xi_{i}}\left(d_{i}(0)-v\right)^{2} \\
& +\frac{1}{2} \sum_{k=1}^{m-1} \sum_{i=1}^{N} \int_{-\tau_{k}}^{0} e_{i}^{T}(s) e_{i}(s) d s .
\end{aligned}
$$

Remark 14. Corollary 13 can be regarded as a special form of Theorem 11 when the time-varying delay $\tau_{k}(t)$ is a constant time delay $\tau_{k}(k=0,1, \ldots, m-1)$ in system (1). It can be also understood as without time-varying. In other words, Corollary 13 makes the complex multilinks networks with 
time delay and perturbations realize the finite-time synchronization in the mean square.

Remark 15. By comparing the controller (14) with the controller (42), we can find that the controller with constant time delay is simpler. If there is no perturbations and delay, the network model and the controller will be simpler, which makes the network structure easier to achieve the synchronization.

\section{Simulation Examples}

In the previous section, we propose criteria for the finitetime synchronization of complex multilinks network with perturbations and time-varying delay via adaptive nonlinear feedback controller (14). In this section, we provide two different numerical simulation examples to verify the effectiveness of our criteria.

Example 16. This example is used to verify the effectiveness of our theoretical results on the finite-time synchronization in complex multilinks networks with constant time delay and perturbations.

This example gives a numerical simulation of Corollary 13. In this example, we consider a network with 8 nodes, and each node is a three-dimensional Lorenz system. Their links have three properties of complex multilinks networks with time delay and perturbations; in other words, $m=3$. The driving network is given as follows:

$$
\begin{aligned}
& d x_{i}(t)=\left[h\left(x_{i}(t)\right)+\sum_{j=1}^{8} c_{(0) i j} x_{j}(t)\right. \\
& \left.+\sum_{j=1}^{8} \mathcal{c}_{(1) i j} x_{j}\left(t-\tau_{1}\right)+\sum_{j=1}^{8} c_{(2) i j} x_{j}\left(t-\tau_{2}\right)\right] d t \\
& +\sigma\left(x_{i}(t), t\right) d \varphi(t), \quad i=1,2, \ldots, 8
\end{aligned}
$$

where

$$
\begin{aligned}
{\left[\begin{array}{l}
h\left(x_{i 1}(t)\right) \\
h\left(x_{i 2}(t)\right) \\
h\left(x_{i 3}(t)\right)
\end{array}\right]=} & {\left[\begin{array}{ccc}
-10 & 10 & 0 \\
28 & -1 & 0 \\
0 & 0 & -\frac{8}{3}
\end{array}\right]\left[\begin{array}{l}
x_{i 1}(t) \\
x_{i 2}(t) \\
x_{i 3}(t)
\end{array}\right] } \\
& +\left[\begin{array}{c}
0 \\
-x_{i 1} x_{i 3} \\
x_{i 1} x_{i 2}
\end{array}\right]
\end{aligned}
$$

$C_{0}, C_{1}$, and $C_{2}$ are the Laplace matrices as well as E$\mathrm{R}$ random network model, and the connection probabilities are $0.8,0.6$, and 0.4 , respectively. They are described as follows:

$$
C_{0}=\left[\begin{array}{cccccccc}
-2 & 0 & 0 & 1 & 0 & 0 & 1 & 0 \\
0 & -4 & 0 & 1 & 1 & 0 & 1 & 1 \\
0 & 0 & -2 & 0 & 0 & 1 & 0 & 1 \\
1 & 1 & 0 & -4 & 1 & 1 & 0 & 0 \\
0 & 1 & 0 & 1 & -3 & 0 & 0 & 1 \\
0 & 0 & 1 & 1 & 0 & -3 & 1 & 0 \\
1 & 1 & 0 & 0 & 0 & 1 & -4 & 1 \\
0 & 1 & 1 & 0 & 1 & 0 & 1 & -4
\end{array}\right],
$$$$
C_{1}=\left[\begin{array}{cccccccc}
-2 & 1 & 0 & 0 & 1 & 0 & 0 & 0 \\
1 & -1 & 0 & 0 & 0 & 0 & 0 & 0 \\
0 & 0 & -1 & 0 & 0 & 1 & 0 & 0 \\
0 & 0 & 0 & -2 & 1 & 0 & 1 & 0 \\
1 & 0 & 0 & 1 & -2 & 0 & 0 & 0 \\
0 & 0 & 1 & 0 & 0 & -1 & 0 & 0 \\
0 & 0 & 0 & 1 & 0 & 0 & -2 & 1 \\
0 & 0 & 0 & 0 & 0 & 0 & 1 & -1
\end{array}\right],
$$$$
C_{2}=\left[\begin{array}{cccccccc}
-3 & 0 & 1 & 0 & 1 & 0 & 0 & 1 \\
0 & -1 & 0 & 0 & 0 & 0 & 1 & 0 \\
1 & 0 & -3 & 0 & 1 & 0 & 0 & 1 \\
0 & 0 & 0 & -2 & 0 & 1 & 1 & 0 \\
1 & 0 & 1 & 0 & -2 & 0 & 0 & 0 \\
0 & 0 & 0 & 1 & 0 & -1 & 0 & 0 \\
0 & 1 & 0 & 1 & 0 & 0 & -3 & 1 \\
1 & 0 & 1 & 0 & 0 & 0 & 1 & -3
\end{array}\right] .
$$

$\tau_{1}=0.04$ and $\tau_{2}=0.1$ are the constant delay-times. And the noise intensity function is given by

$$
\sigma\left(x_{i}(t, t)\right)=\left[\begin{array}{l}
\sigma\left(x_{i 1}(t)\right) \\
\sigma\left(x_{i 2}(t)\right) \\
\sigma\left(x_{i 3}(t)\right)
\end{array}\right]=\left[\begin{array}{l}
0.1 x_{i 1}(t) \\
0.3 x_{i 2}(t) \\
0.5 x_{i 3}(t)
\end{array}\right] .
$$

Correspondingly, the response system with adaptive controller (14) can be described as follows:

$$
\begin{aligned}
& d y_{i}(t)=\left[h\left(y_{i}(t)\right)+\sum_{j=1}^{8} \mathcal{c}_{(0) i j} y_{j}(t)\right. \\
& \left.+\sum_{j=1}^{8} \mathcal{c}_{(1) i j} y_{j}\left(t-\tau_{1}\right)+\sum_{j=1}^{8} c_{(2) i j} y_{j}\left(t-\tau_{2}\right)\right] d t \\
& +\sigma\left(y_{i}(t), t\right) d \varphi(t)+u_{i}(t) d t
\end{aligned}
$$




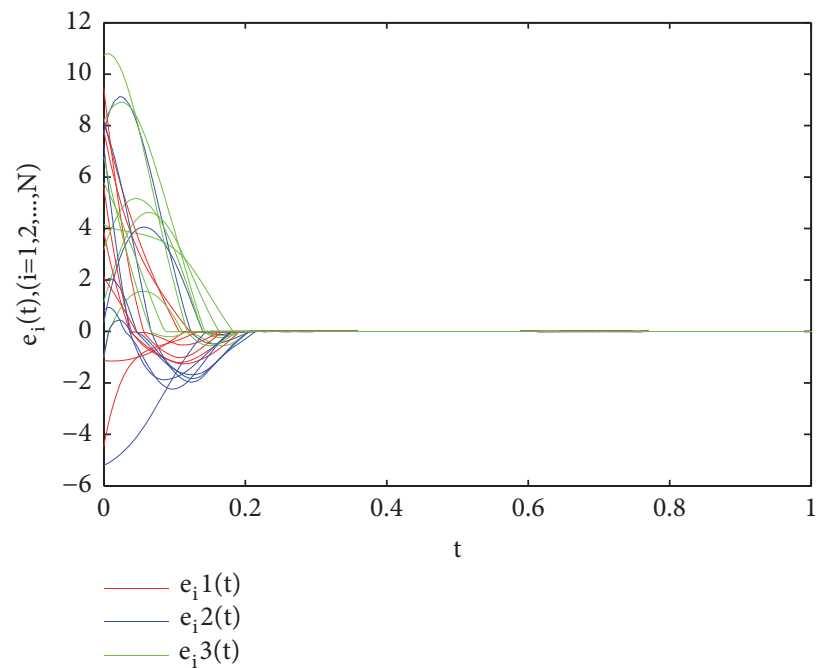

FIgURE 2: The synchronization errors $e_{i}(t)(1 \leqslant i \leqslant 8)$ between the driving and the response networks with constant delay-time in Example 16.

where $y_{i}(t)$ is also the node of the three-dimensional Lorenz system. The noise intensity function is given as follows:

$$
\sigma\left(y_{i}(t, t)\right)=\left[\begin{array}{l}
\sigma\left(y_{i 1}(t)\right) \\
\sigma\left(y_{i 2}(t)\right) \\
\sigma\left(y_{i 3}(t)\right)
\end{array}\right]=\left[\begin{array}{l}
0.1 y_{i 1}(t) \\
0.3 y_{i 2}(t) \\
0.5 y_{i 3}(t)
\end{array}\right] .
$$

In this simulation, we set up the simulation time $t \epsilon$ $[0,1]$. The values of the parameters for the nonlinear adaptive feedback controller (42) are taken as $p=20, q=20, r=$ $20, \xi_{i}=1$ based on Corollary 13. Using the LMI toolbox, we calculate that $v \geq 22.5$ can satisfy inequality (44) in Corollary 13; then we take $v=25$. Through the calculation, we can get the following results. For the first dimension, we get the settling time $T_{s}=0.5222$. For the second dimension, we obtain $T_{s}=0.5434$. For the third dimension, we have $T_{s}=$ 0.6669 . Figures 2-3 show the time response curves of the synchronization errors $e_{i}(t)$ and the separate synchronous errors $e_{i} 1(t), e_{i} 2(t), e_{i} 3(t) \quad(1 \leqslant i \leqslant 8)$ between the driving and the response networks with constant delay-time under the controller (42), respectively. From Figures 2-3, it can be seen that the actual synchronization times are shorter than the theoretical results. The above simulation results demonstrate the validity of Corollary 13.

Example 17. This example is used to verify the effectiveness of our theoretical results on the finite-time synchronization in complex multilinks networks with perturbations and timevarying delay.

This example provides a numerical simulation of Theorem 11. In this example, there are five nodes in the complex multilinks networks, and each node is a three-dimensional Lorenz system. There are two links between each pair of nodes; that is, $m=2$. The driving network is described as follows:
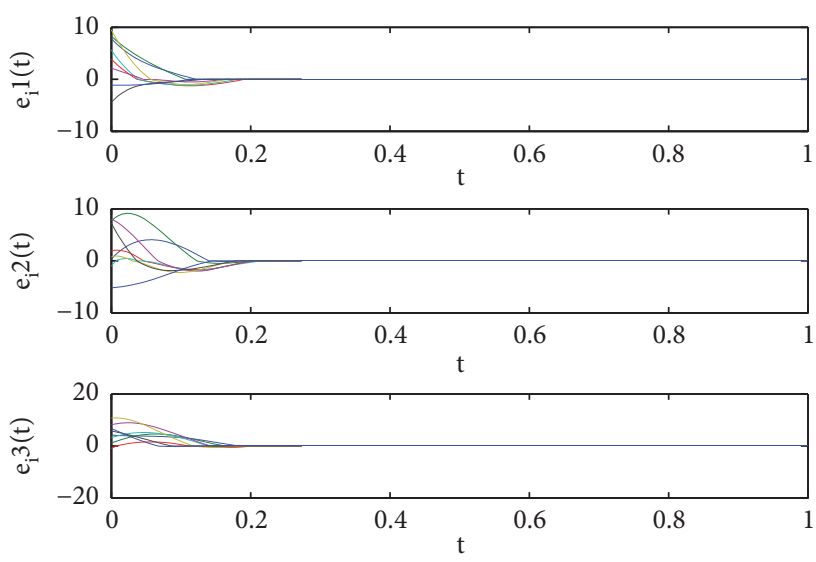

FIGURE 3: The separate synchronous errors $e_{i} 1(t), e_{i} 2(t), e_{i} 3(t)(1 \leqslant$ $i \leqslant 8$ ) between the driving and response networks with constant delay-time in Example 16.

$$
\begin{array}{r}
d x_{i}(t)=\left[h\left(x_{i}(t)\right)+\sum_{j=1}^{5} c_{(0) i j} x_{j}(t)\right. \\
\left.+\sum_{j=1}^{5} c_{(1) i j} x_{j}\left(t-\tau_{1}(t)\right)\right] d t+\sigma\left(x_{i}(t), t\right) d \varphi(t), \\
i=1,2, \ldots, 5 .
\end{array}
$$

The definitions of $h\left(x_{i}(t)\right)$ and $\sigma\left(x_{i}(t), t\right)$ are the same as those in Example 16. $C_{0}=\left(c_{(0) i j}\right)_{5 \times 5}$ is a small-world network model, and the rewiring probability among the nodes is 0.4 . $C_{1}=\left(c_{(1) i j}\right)_{5 \times 5}$ is an E-R random network model, and the connection probability is 0.8 . They are also Laplace matrices described as follows:

$$
\begin{aligned}
C_{0} & =\left[\begin{array}{ccccc}
-1 & 0 & 0 & 0 & 1 \\
0 & -1 & 0 & 0 & 1 \\
0 & 0 & -2 & 1 & 1 \\
0 & 0 & 1 & -1 & 0 \\
1 & 1 & 1 & 0 & -3
\end{array}\right], \\
C_{1} & =\left[\begin{array}{ccccc}
-2 & 0 & 1 & 1 & 0 \\
0 & -1 & 1 & 0 & 0 \\
1 & 1 & -3 & 0 & 1 \\
1 & 0 & 0 & -2 & 1 \\
0 & 0 & 1 & 1 & -2
\end{array}\right] .
\end{aligned}
$$

$\tau_{1}(t)=e^{t} /\left(2+e^{t}\right)$ is the time-varying coupling delay, and we take $\varepsilon=1 / 2$. Then we get $\dot{\tau}_{1}(t)=2 e^{t} /\left(2+e^{t}\right)^{2} \in(0,1 / 4]$, which satisfies Assumption 3.

Correspondingly, the response system with adaptive controller (14) can be described as follows: 


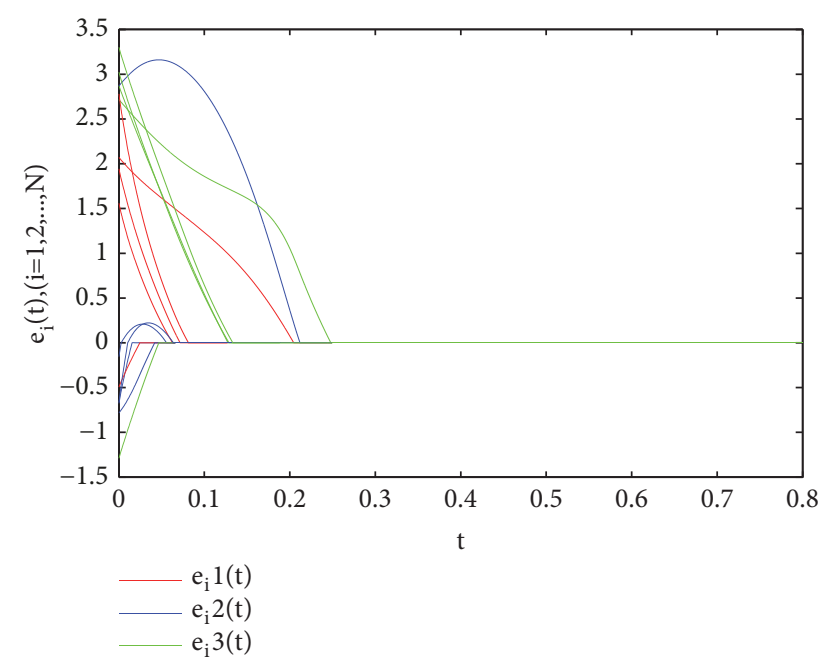

FIgURE 4: The synchronization errors $e_{i}(t)(1 \leqslant i \leqslant 5)$ between the driving and response networks with time-varying delay for different network models in Example 17, where $C_{0}$ is the small-world network model and $C_{1}$ is the random network model.

$$
\begin{aligned}
& d y_{i}(t)=\left[h\left(y_{i}(t)\right)+\sum_{j=1}^{5} c_{(0) i j} y_{j}(t)\right. \\
& \left.+\sum_{j=1}^{5} c_{(1) i j} y_{j}\left(t-\tau_{1}(t)\right)\right] d t+\sigma\left(y_{i}(t), t\right) d \varphi(t) \\
& +u_{i}(t) d t, \quad i=1,2, \ldots, 5
\end{aligned}
$$

where $y_{i}(t)$ is also the node of the three-dimensional Lorenz system. The definition of $\sigma\left(y_{i}(t), t\right)$ is the same as that in Example 16.

In this simulation, the simulation time is set as $t \in[0,0.8]$. The values of the parameters for the nonlinear adaptive feedback controller (14) are taken as $p=15, q=10, r=10$, $\xi_{i}=1$ based on Theorem 11. On the basis of LMI toolbox, $v \geq$ 20 can satisfy inequality (17) in Theorem 11; then we take $\nu=$ 23. Through the calculation, we can get the following results. For the first dimension, we get $T_{s}=0.3893$. For the second dimension, we obtain $T_{s}=0.4336$. For the third dimension, we have $T_{s}=0.6719$. Figures $4-5$ show the time response curves of the synchronization errors $e_{i}(t)$ and the separate synchronous errors $e_{i} 1(t), e_{i} 2(t), e_{i} 3(t)(1 \leqslant i \leqslant 5)$ between the driving and response networks with time-varying delay under the controller (14), respectively. From Figures 4-5, it can be seen that the actual synchronization times are shorter than the theoretical results. Figures 4 and 6-8 present the curves of synchronization errors for different networks modes. By comparison, we can find that different networks have different convergence speeds and vibration extents of synchronous errors, but they all achieve the synchronization in the finite time. Therefore, Theorem 11 is widely applicable to subnetworks with various properties. The simulation results verify the feasibility of the proposed theorem.
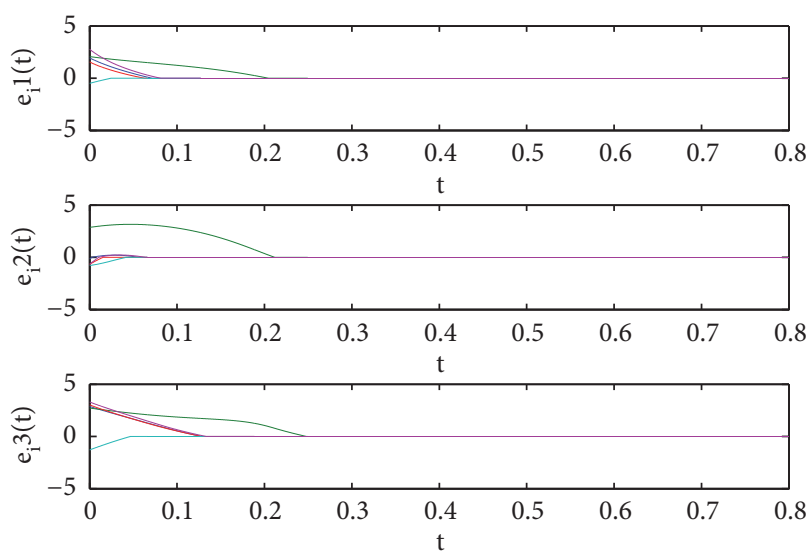

FIGURE 5: The separate synchronous errors $e_{i} 1(t), e_{i} 2(t), e_{i} 3(t)(1 \leqslant$ $i \leqslant 5$ ) between the driving and the response networks with timevarying delay for different network models in Example 17, where $C_{0}$ is the small-world network model and $C_{1}$ is the random network model.

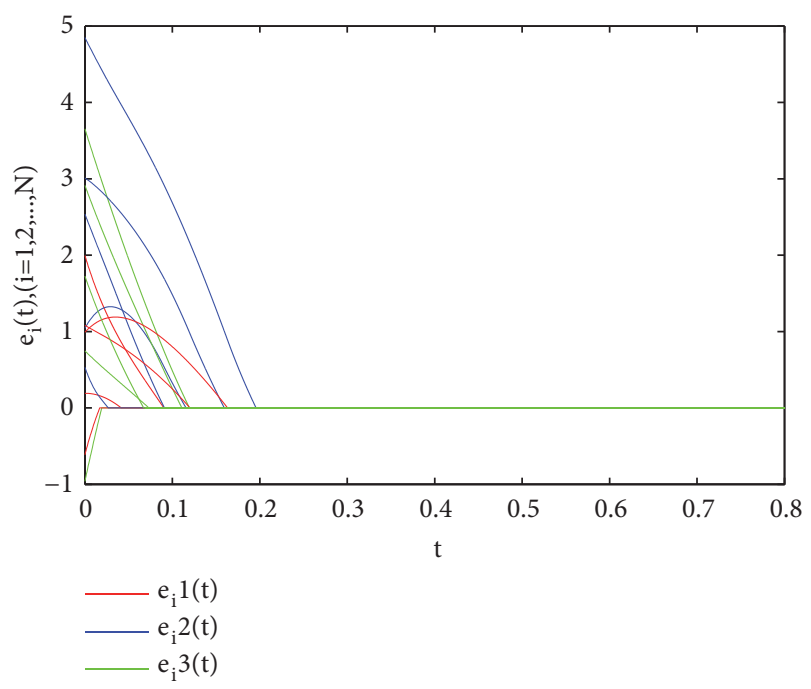

FIgURE 6: The synchronization errors $e_{i}(t)(1 \leqslant i \leqslant 5)$ for the network models in Example 17, where $C_{0}$ and $C_{1}$ are all random network models.

\section{Conclusions}

This paper designed an adaptive nonlinear feedback controller to achieve the finite-time synchronization of the complex multilinks networks with perturbations and timevarying delay. Based on the finite-time stability theory, we successfully ensured the finite-time synchronization between the drive system and the response system in terms of adaptive controls. Some novel adaptive control laws and useful finitetime synchronization criteria were rigorously derived by linear matrix inequality, Kronecker product and several inequality analytical techniques. Finally, both numerical simulation examples and theoretical analyses were investigated to verify the validity and the effectiveness of our results. 


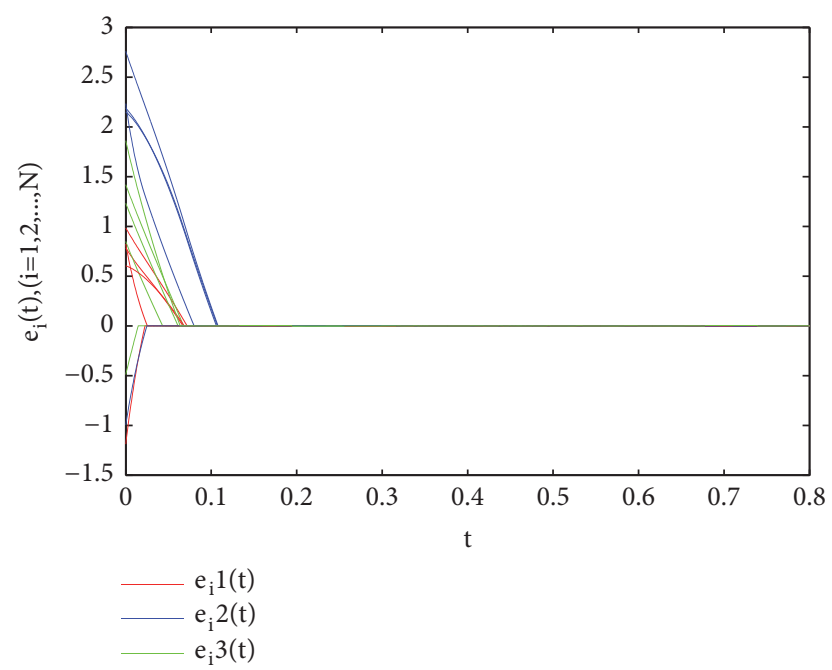

FIgURE 7: The synchronization errors $e_{i}(t)(1 \leqslant i \leqslant 5)$ for different network models in Example 17, where $C_{0}$ is the random network model and $C_{1}$ is the B-A scale-free network model.

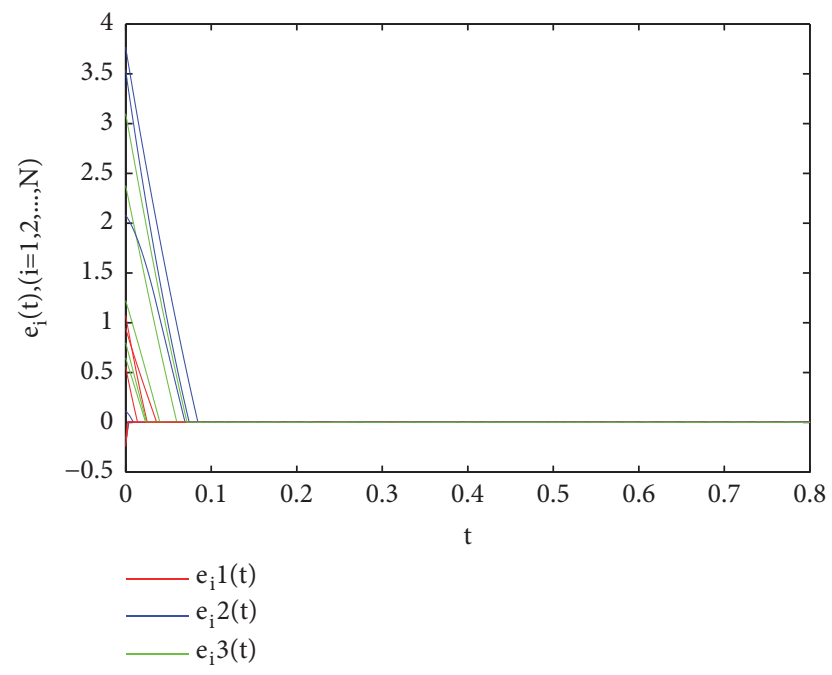

FIGURE 8: The synchronization errors $e_{i}(t)(1 \leqslant i \leqslant 5)$ for different network models in Example 17, where $C_{0}$ is the small-world network model and $C_{1}$ is the B-A scale-free network model.

\section{Data Availability}

The data used to support the findings of this study are included within the article.

\section{Conflicts of Interest}

The authors declare no conflicts of interest.

\section{Acknowledgments}

The work is supported by the National Key R\&D Program of China (Grant nos. 2016YFB0800604 and 2016YFB0800602), the National Natural Science Foundation of China (Grant nos. 61573067, 61771071), and the "13th Five-Year"
National Crypto Development Fund of China (Grant no. MMJJ20170122).

\section{References}

[1] M. Faloutsos, P. Faloutsos, and C. Faloutsos, "On power-law relationships of the internet topology," in Proceedings of the ACM Conference on Applications, Technologies, Architectures, and Protocols for Computer Communication (SIGCOMM '99), vol. 29, pp. 251-262, 1999.

[2] E. Ravasz, A. L. Somera, D. A. Mongru, Z. N. Oltvai, and A. L. Barabási, "Hierarchical organization of modularity in metabolic networks," Science, vol. 297, no. 5586, pp. 1551-1555, 2002.

[3] M. Chavez, M. Valencia, V. Latora, and J. Martinerie, "Complex networks: New trends for the analysis of brain connectivity," International Journal of Bifurcation and Chaos, vol. 20, no. 6, pp. 1677-1686, 2010.

[4] R. Albert, H. Jeong, and A.-L. Barabasi, "Error and attack tolerance of complex networks," Nature, vol. 406, no. 4, pp. 378$382,2000$.

[5] D. J. Watts, P. S. Dodds, and M. E. J. Newman, "Identity and search in social networks," Science, vol. 296, no. 5571, pp. 1302 1305, 2002.

[6] R. Rakkiyappan and K. Sivaranjani, "Sampled-data synchronization and state estimation for nonlinear singularly perturbed complex networks with time-delays," Nonlinear Dynamics, vol. 84, no. 3, pp. 1623-1636, 2016.

[7] S. Abe and N. Suzuki, "Scale-free network of earthquakes," EPL (Europhysics Letters), vol. 65, no. 4, pp. 581-586, 2004.

[8] S. Lozano, L. Buzna, and A. Díaz-Guilera, "Role of network topology in the synchronization of power systems," The European Physical Journal B, vol. 85, pp. 1-8, 2012.

[9] H. Jeong, B. Tombor, R. Albert, Z. N. Oltval, and A.-L. Barabásl, "The large-scale organization of metabolic networks," Nature, vol. 407, no. 6804, pp. 651-654, 2000.

[10] B. Stefan and G. S. Hans, Handbook of Graphs and Networks: From the Genome to the Internet, Germany:Wiley-VCH, 2003.

[11] H. Ebel, L.-I. Mielsch, and S. Bornholdt, "Scale-free topology of e-mail networks," Physical Review E: Statistical, Nonlinear, and Soft Matter Physics, vol. 66, no. 3, Article ID 035103, 2002.

[12] S. A. Plotnikov, J. Lehnert, A. L. Fradkov, and E. Scholl, "Control of synchronization in delay-coupled neural networks of heterogeneous nodes," International Journal of Bifurcation \& Chaos, vol. 23, pp. 435-455, 2015.

[13] F. Sorrentino, L. M. Pecora, A. M. Hagerstrom, T. E. Murphy, and R. Roy, "Complete characterization of the stability of cluster synchronization in complex dynamical networks," Science Advances, vol. 2, no. 4, p. e1501737, 2016.

[14] M. Zheng, L. Li, H. Peng, J. Xiao, Y. Y. Yang, and H. Zhao, "Parameters estimation and synchronization of uncertain coupling recurrent dynamical neural networks with time-varying delays based on adaptive control," Neural Computing \& Applications, pp. 1-11, 2016.

[15] J. Yu, C. Hu, H. Jiang, and Z. Teng, "Stabilization of nonlinear systems with time-varying delays via impulsive control," Neurocomputing, vol. 125, no. 3, pp. 68-71, 2014.

[16] F. Wang, Y. Yang, M. Hu, and X. Xu, "Projective cluster synchronization of fractional-order coupled-delay complex network via adaptive pinning control," Physica A: Statistical Mechanics and its Applications, vol. 434, pp. 134-143, 2015. 
[17] J.-A. Wang, X. Ma, X. Wen, and Q. Sun, "Pinning lag synchronization of drive-response complex networks via intermittent control with two different switched periods," Physica A: Statistical Mechanics and its Applications, vol. 461, pp. 278-287, 2016.

[18] S. M. Tabatabaei and M. M. Arefi, "Adaptive neural control for a class of uncertain non-affine nonlinear switched systems," Nonlinear Dynamics, vol. 83, no. 3, pp. 1773-1781, 2016.

[19] P. DeLellis, M. diBernardo, and F. Garofalo, "Novel decentralized adaptive strategies for the synchronization of complex networks," Automatica, vol. 45, no. 5, pp. 1312-1318, 2009.

[20] W. Yu, P. DeLellis, G. Chen, M. di Bernardo, and J. Kurths, "Distributed adaptive control of synchronization in complex networks," IEEE Transactions on Automatic Control, vol. 57, no. 8, pp. 2153-2158, 2012.

[21] F. Nian, "Adaptive coupling synchronization in complex network with uncertain boundary," Nonlinear Dynamics, vol. 70, no. 1, pp. 861-870, 2012.

[22] Y. Lu, P. He, S.-H. Ma, G.-Z. Li, and S. Mobayben, "Robust adaptive synchronization of general dynamical networks with multiple delays and uncertainties," Pramana-Journal of Physics, vol. 86, no. 6, pp. 1223-1241, 2016.

[23] J. M. Montoya and R. V. Solé, "Small world patterns in food webs,” Journal of Theoretical Biology, vol. 214, no. 3, pp. 405-412, 2002.

[24] M. E. J. Newman, "The structure and function of complex networks," SIAM Review, vol. 45, no. 2, pp. 167-256, 2003.

[25] R. Albert and A. Barabási, "Statistical mechanics of complex networks," Reviews of Modern Physics, vol. 74, no. 1, pp. 47-97, 2002.

[26] H. Peng, N. Wei, L. Li, W. Xie, and Y. Yang, "Models and synchronization of time-delayed complex dynamical networks with multi-links based on adaptive control," Physics Letters A, vol. 374, no. 23, pp. 2335-2339, 2010.

[27] M. Jiang, S. Fang, and X. Wang, "Adaptive synchronization of complex dynamical networks with coupling delays and multilinks," in Proceedings of the International Workshop on Advanced Computational Intelligence, p. 374, IEEE, 2010.

[28] W. Wang, L. Li, H. Peng, J. Yuan, J. Xiao, and Y. Yang, "Adaptive synchronization of complex dynamical multilinks networks with similar nodes," Mathematical Problems in Engineering, vol. 2013, Article ID 736585, 244 pages, 2013.

[29] M. Zheng, L. Li, H. Peng et al., "Finite-time synchronization of complex dynamical networks with multi-links via intermittent controls," The European Physical Journal B, vol. 89, pp. 1-12, 2016.

[30] P. Erdös and A. Rényi, "On the evolution of random graphs," Publications of the Mathematical Institute of the Hungarian Academy of Sciences, vol. 5, pp. 17-61, 1960.

[31] A. Barabasi and R. Albert, "Emergence of scaling in random networks," Science, vol. 286, no. 5439, pp. 509-512, 1999.

[32] D. J. Watts and S. H. Strogatz, "Collective dynamics of "smallworld” networks," Nature, vol. 393, no. 6684, pp. 440-442, 1998.

[33] S. P. Bhat and D. S. Bernstein, "Finite-time stability of continuous autonomous systems," SIAM Journal on Control and Optimization, vol. 38, no. 3, pp. 751-766, 2000.

[34] S. Boyd, L. El Ghaoui, E. Feron, and V. Balakrishnan, Linear Matrix Inequalities in System and Control Theory, SIAM, Philadelphia, Pa, USA, 1994.

[35] X. Liu, X. Yu, and H. Xi, "Finite-time synchronization of neutral complex networks with Markovian switching based on pinning controller," Neurocomputing, vol. 153, pp. 148-158, 2015.
[36] X.-Z. Jin and G.-H. Yang, "Robust synchronization control for complex networks with disturbed sampling couplings," Communications in Nonlinear Science and Numerical Simulation, vol. 19, no. 6, pp. 1985-1995, 2014. 


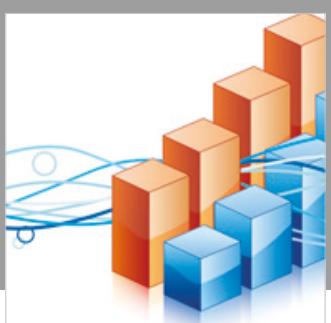

Advances in

Operations Research

\section{-n-m}
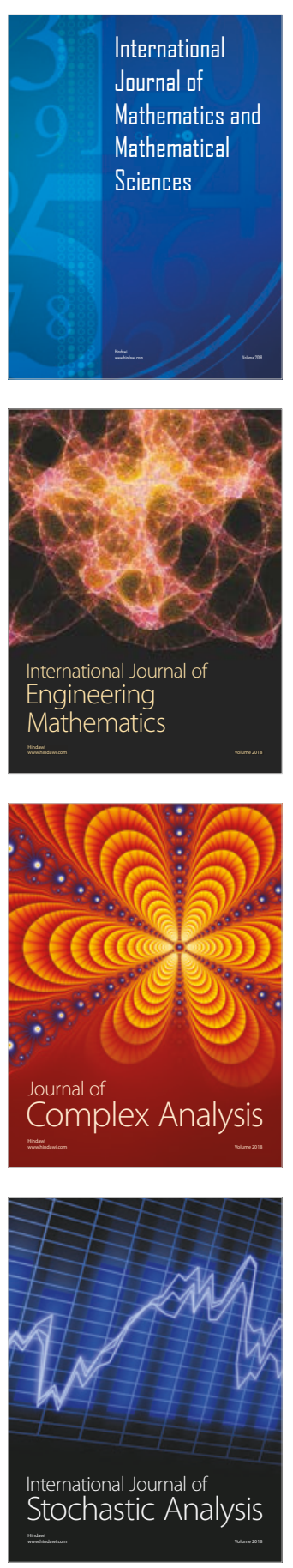
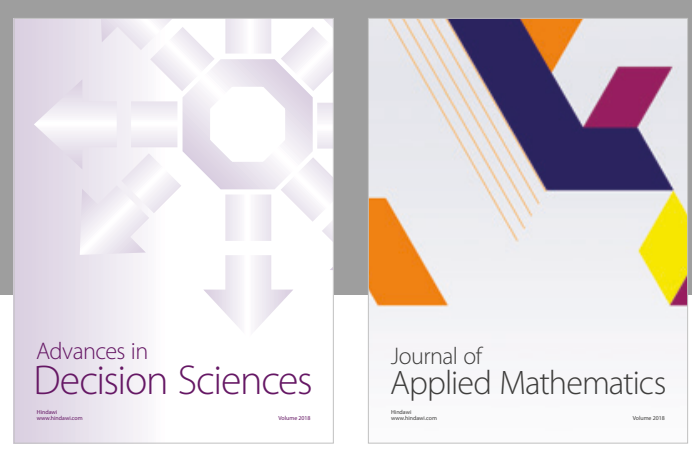

Journal of

Applied Mathematics
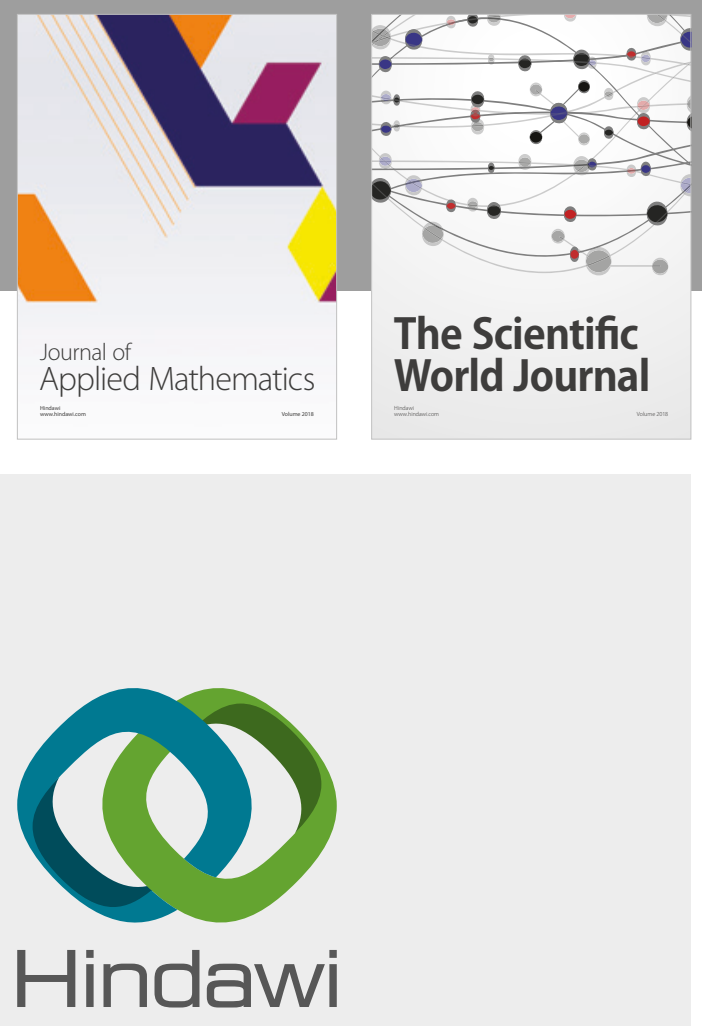

Submit your manuscripts at

www.hindawi.com

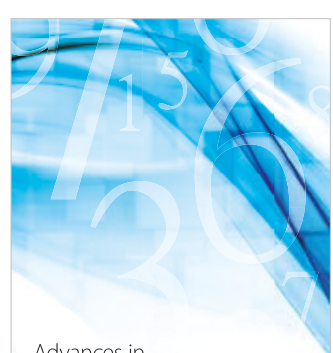

Advances in
Numerical Analysis
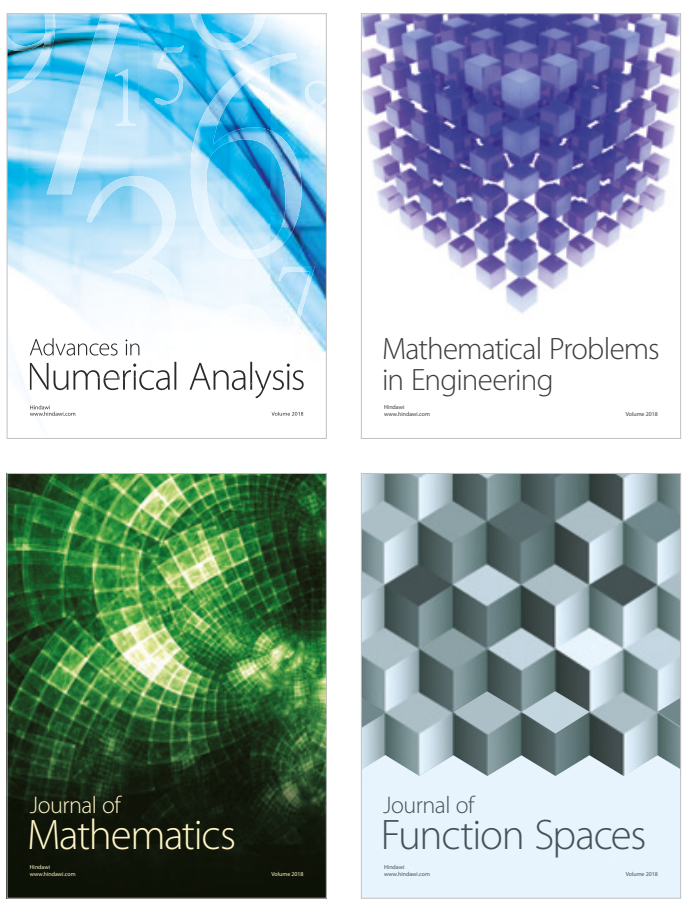

Mathematical Problems in Engineering

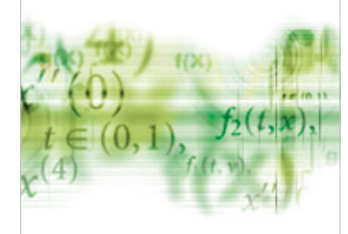

International Journal of

Differential Equations

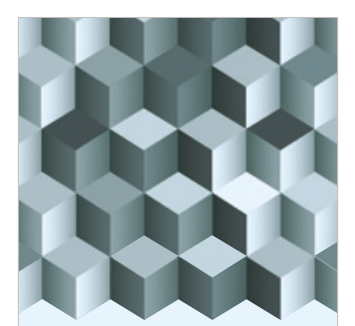

Journal of

Function Spaces

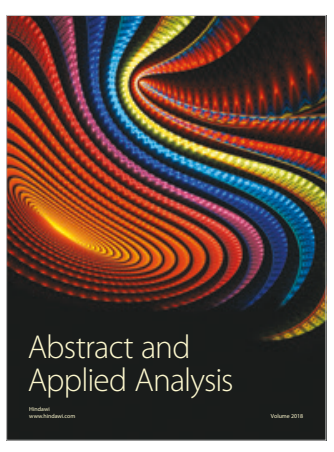

The Scientific

World Journal

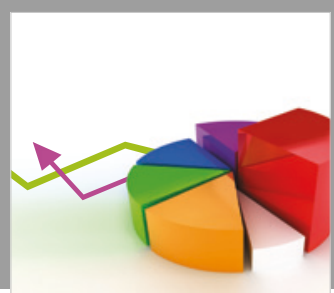

Journal of

Probability and Statistics
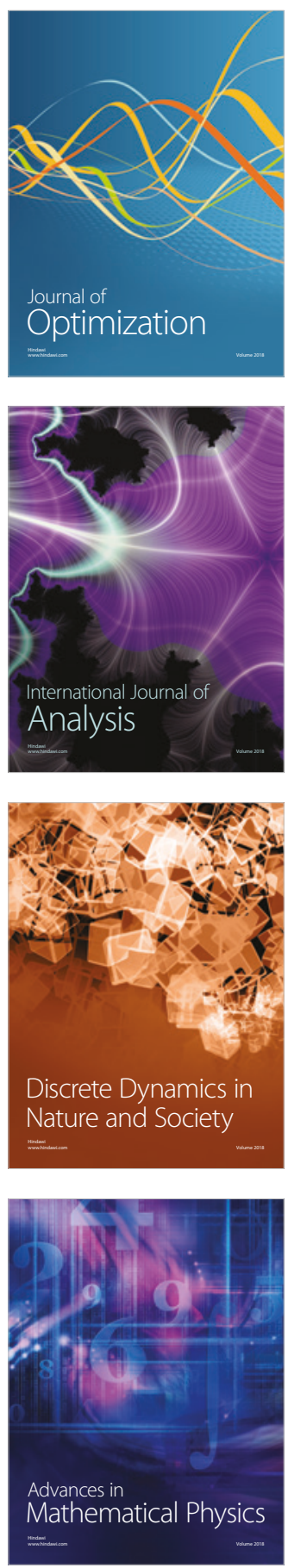